\title{
Evaluación productiva y reproductiva de cuatro genotipos lecheros en pastoreo libre en la amazonía ecuatoriana
}

\author{
Quinteros-Pozo, R. ${ }^{1,3}$; Marini, P.R. ${ }^{2,3}$ \\ ${ }^{1}$ Univ.Estatal Amazónica, Fac.Cs.Agrop., Paso Lateral Km 2 1/2 Vía Napo Puyo, Pastaza, Ecuador. \\ ${ }^{2}$ Fac.Cs.Vet.UNR, Ovidio Lagos y Ruta 33, Casilda, Provincia Santa Fe, Argentina. \\ ${ }^{3}$ Centro Lat. Estudios Problem. Lecheras. E-mail: pmarini@fveter.unr.edu.ar
}

\begin{abstract}
Resumen
Quinteros-Pozo, R.; Marini, P.R.: Evaluación productiva y reproductiva de cuatro genotipos lecheros en pastoreo libre en la amazonía ecuatoriana. Rev. vet. 28: 1, 9-13, 2017. El objetivo de este estudio fue evaluar el comportamiento productivo y reproductivo de cuatro genotipos lecheros en condiciones de pastoreo libre en el Cantón Arosemena Tola, Provincia de Napo, Ecuador. Se evaluaron 36 vacas lecheras de primer parto, cruzas de diferentes genotipos: Brahman x Gyrolando, Brahman x Brown Swiss, Brahman x Jersey y Brahman x Sahiwall. No se registraron diferencias significativas $(p \geq 0,05)$ entre las variables productivas estudiadas de los cuatro genotipos empleados. Tampoco existieron diferencias significativas entre los pesos al parto para los cuatro genotipos, sin embargo, adquirieron significación estadística a los 90 días posparto y al secado $(\mathrm{p} \leq 0,001)$. Por el contrario, en las variables reproductivas analizadas existieron diferencias significativas entre los cuatro genotipos a la edad del primer parto $(\mathrm{p} \leq 0,001)$. Se concluye que los cuatro genotipos lecheros estudiados se comportan de forma similar acorde a la región en estudio. Ninguno de los genotipos lecheros analizados se destacó por encima del otro.
\end{abstract}

Palabras clave: bovinos lecheros, genotipos, pastoreo, producción, reproducción.

\begin{abstract}
Abstrac
Quinteros-Pozo, R.; Marini, P.R.: Productive and reproductive evaluation of four dairy genotypes under free grazing in the Ecuadorian Amazonia. Rev. vet. 28: 1, 9-13, 2017. The aim of this study was to evaluate the productive and reproductive performance of four dairy genotypes under free grazing in Canton Arosemena Tola, Napo Province, Ecuador. Thirty six dairy cows of first birth and different cross genotypes: Brahman x Gyrolando, Brahman $\mathrm{x}$ Brown Swiss, Brahman x Jersey and Brahman x Sahiwall, were evaluated. The production variables under consideration did not reveal significant differences $(\mathrm{p} \geq 0.05)$ among the four genotypes. The analyzed reproductive variables showed significant differences $(p \leq 0.001)$ among the four genotypes studied at the age of first birth. There were also no significant differences ( $\mathrm{p} \geq 0.05)$ in delivery weights for the four genotypes, however statistical differences were found in weights at 90 days postpartum $(\mathrm{p} \leq 0.01)$ and drying $(\mathrm{p} \leq 0.001)$. It is concluded that the four genotypes behave similarly regarding the region under consideration. None of the genotypes stood out above the other, showing parity among them.
\end{abstract}

Key words: dairy cattle, genotypes, grazing system, production, reproduction.

\section{INTRODUCCIÓN}

$\mathrm{El}$ arribo de animales de razas especializadas a las regiones tropicales no ha tenido éxito, siendo algunas de las causas: los problemas de salud y adaptación, diferentes objetivos de producción entre las regiones de origen y las regiones tropicales, así como escasez o ausencia de la infraestructura requerida para la reco-

Recibido: 10 junio 2016 / Aceptado: 3 noviembre 2016 lección y procesamiento de la información producida. Además, los costos de mantenimiento y producción con razas especializadas generan menores márgenes de ganancia e inclusive pérdidas en comparación de los sistemas tradicionales de producción a pastoreo ${ }^{13,14}$.

Las estrategias de cruzamiento, selección y mejoramiento en sistemas de producción de ganado bovino en los trópicos, deben formularse teniendo en cuenta el agroecosistema y los aspectos económicos, ambientales y socio-culturales, haciendo énfasis en las caracte- 
rísticas de rendimiento y reproducción, con el fin de lograr eficiencia en la producción sostenible del sistema ${ }^{4}$.

El cruzamiento de ganado lechero se ha convertido en un tema de considerable interés en respuesta a las preocupaciones de los productores de leche por la disminución de la fertilidad, la salud y la supervivencia de vacas especializadas. En los cruzamientos, los productores tienen como objetivo explotar las características favorables de las razas alternativas, eliminar los efectos negativos asociados con la endogamia y sacar provecho de la heterosis. Resultados de investigaciones recientes ilustran claramente que es posible lograr mayor fertilidad y supervivencia en las vacas cruzas ${ }^{3}$.

En el sistema de producción de bovinos de doble propósito predominante en la región tropical húmeda de México, la producción diaria de leche promedio por vaca es de 3 a $9 \mathrm{~kg}$, la duración de la lactación es de alrededor de 120 a 180 días y los partos se suceden cada 18 a 24 meses ${ }^{15}$.

El objetivo de este trabajo fue generar datos capaces de evaluar y mejorar el comportamiento productivo y reproductivo de cuatro genotipos lecheros de primera lactancia en condiciones de pastoreo libre en la Amazonia Ecuatoriana, Cantón Arosemena Tola, Provincia de Napo, Ecuador.

\section{MATERIAL Y MÉTODOS}

Diseño y población de estudio. Se evaluó el comportamiento productivo y reproductivo de 36 vacas lecheras cruzas, de primer parto, de cuatro genotipos: Brahman x Gyrolando (G), Brahman x Brown Swiss (B), Brahman x Jersey (J) y Brahman x Sahiwall (S) pertenecientes al rodeo lechero del Centro de Investigación, Posgrado y Conservación de la Biodiversidad Amazónica (CIPCA). Dicho centro está ubicado en el cantón Arosemena Tola de la Provincia de Napo (Ecuador), en el kilómetro 44 vía Puyo-Tena (coordenadas: $\mathrm{S}$ $01^{\circ} 14.325^{\prime} ; \mathrm{W} 077^{\circ} 53.134^{\prime}$ ) y dispone de una superficie de 42 ha de pastos destinada a la lechería. El ambiente es tropical con precipitaciones de $4000 \mathrm{~mm} / \mathrm{año}$, humedad relativa promedio del $80 \%$ y temperaturas que varían entre 15 y $25^{\circ} \mathrm{C}$. Su topografía se caracteriza por relieves ligeramente ondulados sin pendientes pronunciadas, distribuidos en mesetas naturales de gran extensión. La altitud varía entre los 580 y $990 \mathrm{msnm}$. Si bien los suelos presentan una composición muy heterogénea, la mayoría se origina en sedimentos fluviales procedentes de la región andina del país. Las vacas evaluadas fueron criadas bajo las mismas condiciones ambientales, nutricionales y de manejo. Los animales ingresaron al CIPCA con 15-17 meses de edad y pesos corporales (promedio \pm error estándar) de $204 \pm 7,7 \mathrm{~kg}(\mathrm{G}) ; 276 \pm$ $11,0 \mathrm{~kg}(\mathrm{~B}) ; 204 \pm 8,7 \mathrm{~kg}(\mathrm{~J})$ y $186 \pm 6,0 \mathrm{~kg}(\mathrm{~S})$.

Manejo. Los datos productivos se obtuvieron del control lechero realizado cada 15 días los primeros 60 días y luego una vez al mes. Las vacas se ordeñaron una vez al día a las 7:00 h, con apoyo de ternero para estimular la eyección de la leche. Los datos reproductivos se tomaron de los controles periódicos efectuados por el asesor veterinario responsable de la lechería del CIPCA. En el lapso de la realización del trabajo, todas los animales se manejaron en la misma instalación de ordeño. Las vacas se inseminaron siempre con semen de toros probados. Se tomaron los pesos de todas las vacas al parto, a los 90 días del parto y al secado; este procedimiento se realizó siempre en el mismo horario (luego del ordeñe). El análisis físico-químico de la leche se realizó durante el control lechero, recolectando $200 \mathrm{ml}$ de leche por vaca, muestras que se depositaron en frascos de vidrio debidamente identificados con el número del animal y el grupo al que pertenecía; posteriormente fueron transportadas y almacenadas a $4^{\circ} \mathrm{C}$ hasta su procesamiento, para determinar densidad y porcentajes de grasa, sólidos totales, sólidos no grasos y proteína, utilizando el analizador de leche ultrasónico Lactoscan ${ }^{\circledR}$ previamente calibrado con métodos estandarizados.

Alimentación y sanidad. La alimentación del hato bovino en estudio se basó en el pastoreo libre sobre pastizales de Brachiaria decumbens (17.585 kg MS/ha/año, proteína $10,6 \%$, fósforo $0,18 \%$, digestibilidad in vivo -DIV- 44,4\%); Brachiaria brizantha (26.970 kg MS/ha/ año, proteína $10,1 \%$, fósforo $0,18 \%$, DIV 44,1\%); Arachis pintoi (6.212 kg MS/ha/año, proteína 19,4\%, fósforo $0,21 \%$, DIV 59,2\%); Desmodium ovalifolium (5.890 $\mathrm{kg} \mathrm{MS} / \mathrm{ha} / \mathrm{año}$, proteína $16,3 \%$, fósforo $0,16 \%$, DIV $39,6 \%)$ y Stylosanthes guianensis (15.237 kg MS/ha/ año, proteína $21,4 \%$, fósforo $0,4 \%$, DIV $48,7 \%$ ). Se aplicó el manejo sanitario habitualmente empleado en el CIPCA, consistente en desparasitaciones, baños contra garrapatas y moscas, vacunaciones para fiebre aftosa, rabia bovina y estomatitis vesicular, así como aplicación inyectable de vitaminas y minerales.

Variables utilizadas. Se controlaron las siguientes variables:

-Producción de leche a la primera lactancia $\left(\mathrm{pl}_{150}\right)$ en litros, ajustada a 150 días: $\sum 1_{c 1}$ x 30,5 donde lc son los litros producidos en el j-ésimo control lechero de la primera lactancia y 30,5 es el número medio de días por mes.

-Producción diaria de leche ( $\mathrm{pl}$ ) en litros: $\mathrm{pl}_{150} / \mathrm{du}-$ ración de la lactancia ajustada.

-Porcentaje de proteína $(\mathrm{Pr})$ : proteína en la leche.

-Producción de $\mathrm{kg}$ de proteína (KP): $\mathrm{kg}$ de proteína total a los 150 días de lactancia.

-Peso (P): peso individual en $\mathrm{kg}$.

-Edad al primer parto (EPP): edad al primer parto en días.

-Intervalo parto primer celo (IPPC): días entre el primer parto y retorno a celo posterior.

-Intervalo parto-concepción (IPC): días entre el primer parto y la concepción posterior.

-Intervalo parto-parto (IPP): días transcurridos entre el primer y segundo parto.

Análisis estadístico. Para cada variable estudiada se estimaron la media aritmética $(\bar{x})$ y el error estándar (EE). Se probó si existían diferencias significativas entre los genotipos mediante la aplicación de análisis de 
la variancia (ANOVA) a un criterio de clasificación y pruebas de comparaciones múltiples de Tukey-Kramer HSD $(p \leq 0,05)$. Los análisis estadísticos fueron realizados utilizando el programa JMP (JMP $\left.{ }^{2}, 2003\right)$ en su versión 5.0 para Windows.

\section{RESULTADOS}

La Tabla 1 indica que para las variables productivas analizadas no se registraron diferencias significativas $(p \geq 0,05)$ entre los cuatro genotipos estudiados.

En la Figura 1 se observa que no existieron diferencias significativas $(p \geq 0,05)$ en los pesos al parto entre los cuatro genotipos, sin embargo se advierte que a los 90 días posparto se registraron diferencias significativas $(p \leq 0,01)$ señalando que $B$ fue la que menos peso perdió y $\mathrm{G}$ la que más peso perdió de los cuatro genotipos desde el parto. Las vacas que se secaron con el menor peso, comparado con el de su propio peso al parto, fueron las razas $S$ y $G$, diferencias que fueron significativas $(p \leq 0,001)$.

En la Figura 2 se observa que de los cuatro genotipos estudiados, los animales de menor porcentaje de pérdida de peso entre el parto y el secado, fueron las vacas B $(28 \mathrm{~kg})$. Las vacas J (44 kg) y G (33 kg) revelaron pérdidas intermedias y las vacas $\mathrm{S}$ registraron los mayores porcentajes de pérdida de peso $(58 \mathrm{~kg})$.

En la Tabla 2 se observa que para las variables reproductivas analizadas existieron diferencias significativas $(p \leq 0,001)$ entre los cuatro genotipos estudiados en la edad al primer parto (EPP), siendo las vacas B las más precoces y las vacas $\mathrm{S}$ las que tardaron en promedio más tiempo para tener su primer parto. El resto de las variables no acusó diferencias significativas $(p \geq 0,05)$ entre los genotipos.

\section{DISCUSIÓN}

En la amazonia ecuatoriana el $82 \%$ de la superficie con uso agropecuario está dedicada a pastizales, lo cual demuestra que la ganadería es uno de los rubros de mayor importancia para la economía campesina ${ }^{7}$. La región amazónica genera el 8\% de la producción total de leche de Ecuador. Los sistemas tropicales ofrecen

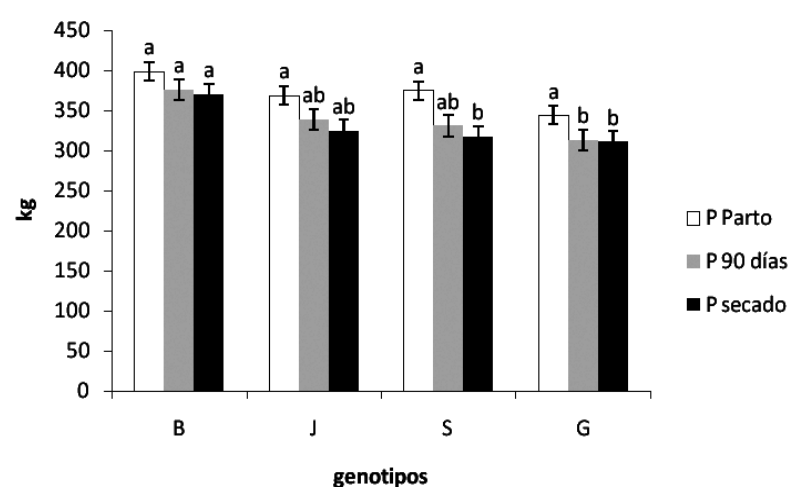

Figura 1. Evolución del peso en los cuatro genotipos. Valores en $\bar{x} \pm E E$; letras distintas indican $p \leq 0,05$.
Tabla 1. Variables productivas para los cuatro genoti$\operatorname{pos}(\overline{\mathrm{X}} \pm \mathrm{EE})$.

\begin{tabular}{ccccc}
\hline gen & $\mathrm{pl}$ (litros) & $\mathrm{pl}_{150}$ (litros) & $\mathrm{P}(\%)$ & $\mathrm{KP}(\mathrm{Kg})$ \\
\hline $\mathrm{B}$ & $6,7 \pm 0,6 \mathrm{a}$ & $1010 \pm 26 \mathrm{a}$ & $3,2 \pm 0,1 \mathrm{a}$ & $28 \pm 3 \mathrm{a}$ \\
$\mathrm{J}$ & $6,6 \pm 0,9 \mathrm{a}$ & $998 \pm 44 \mathrm{a}$ & $3,2 \pm 0,1 \mathrm{a}$ & $21 \pm 3 \mathrm{a}$ \\
$\mathrm{S}$ & $6,6 \pm 0,9 \mathrm{a}$ & $1003 \pm 55 \mathrm{a}$ & $3,3 \pm 0,1 \mathrm{a}$ & $23 \pm 3 \mathrm{a}$ \\
$\mathrm{G}$ & $6,5 \pm 0,7 \mathrm{a}$ & $983 \pm 42 \mathrm{a}$ & $3,3 \pm 0,1 \mathrm{a}$ & $21 \pm 3 \mathrm{a}$ \\
\hline
\end{tabular}

gen: genotipo, pl: producción diaria de leche, $\mathrm{pl}_{150}$ : producción de leche a la primera lactancia, P: proteína láctea, KP: producción de proteína a los 150 días de lactancia. Letras diferentes en una misma columna indican $\mathrm{p} \leq 0,05$.

Tabla 2. Variables reproductivas para los cuatro genotipos $(\bar{x} \pm E E)$.

\begin{tabular}{ccccc}
\hline gen & EPP (días) & IPPC (días) & IPC (días) & IPP (días) \\
\hline $\mathrm{B}$ & $1132 \pm 38 \mathrm{~b}$ & $78 \pm 8 \mathrm{a}$ & $228 \pm 32 \mathrm{a}$ & $509 \pm 32 \mathrm{a}$ \\
$\mathrm{J}$ & $1210 \pm 30 \mathrm{ab}$ & $77 \pm 8 \mathrm{a}$ & $219 \pm 18 \mathrm{a}$ & $500 \pm 18 \mathrm{a}$ \\
$\mathrm{S}$ & $1301 \pm 32 \mathrm{a}$ & $78 \pm 10 \mathrm{a}$ & $162 \pm 34 \mathrm{a}$ & $443 \pm 34 \mathrm{a}$ \\
$\mathrm{G}$ & $1262 \pm 55 \mathrm{ab}$ & $66 \pm 12 \mathrm{a}$ & $215 \pm 64 \mathrm{a}$ & $496 \pm 64 \mathrm{a}$ \\
\hline
\end{tabular}

gen: genotipo, EPP: edad al primer parto, IPPC: intervalo parto-primer celo, IPC: intervalo parto-concepción; IPP: intervalo parto-parto. Letras diferentes en una misma columna indican $\mathrm{p} \leq 0,001$.

ventajas comparativas, la estrategia se basa fundamentalmente en el aprovechamiento de la abundante energía solar disponible para una fotosíntesis eficiente por parte de especies forrajeras megatérmicas ("C4") de rápido crecimiento, así como en la disponibilidad de agua ${ }^{9}$.

En Ecuador, al igual que en muchos países del trópico y subtrópico en general, ha existido una fuerte tendencia a la sustitución de las razas locales por razas puras exóticas, con el fin de incrementar los niveles de producción de leche. Estas razas incorporadas provenían de regiones donde las condiciones climáticas y nutricionales eran más favorables que las imperantes bajo condiciones del trópico, por lo cual su rendimiento disminuyó. Una alternativa a la cual ha recurrido el productor es la utilización de cruzas entre razas cebuinas y razas europeas con el fin de minimizar los problemas de adaptación de las razas puras ${ }^{17}$.

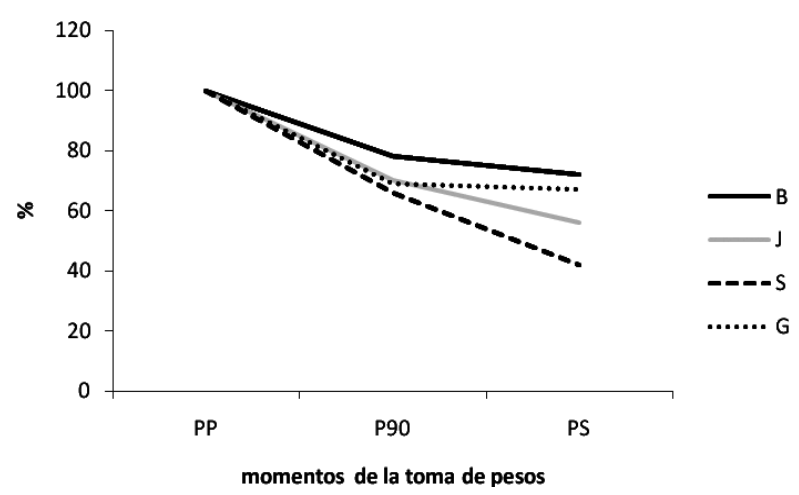

Figura 2. Porcentaje de pérdida de peso de los cuatro genotipos al parto (PP), a los 90 días (P90) y al secado (PS). 
En este trabajo todos los genotipos evaluados mostraron en general similar comportamiento (Tablas $1 \mathrm{y}$ 2). Con respecto a la producción de leche, en las ganaderías de doble propósito tropical, el promedio de producción es de 5 litros por vaca por día ${ }^{11}$. Este dato coincide con los resultados de vacas lecheras cruzas en su primer parto con 305 días de lactancia ajustada, a saber: Brahman (5,4 litros/día con un total de 1566 litros), Criollo (5,5 litros/día y 1684 litros totales), Holstein (6,4 litros/día y 1958 litros totales) y Brown Swiss (5,8 litros/día y 1767 litros totales) ${ }^{1}$.

Los genotipos estudiados en este trabajo mostraron una mayor producción diaria que la anteriormente citada para Brahman, Criollo y Brown Swiss o similares a los encontrados en ese mismo trabajo para Holstein. Seguramente, mejorando la calidad y cantidad de la dieta ofrecida, se podría elevar el promedio de litros por día, pero la realidad indica que ello no siempre es posible y se debería pensar en alternativas con los elementos que se encuentran a disposición.

En los porcentajes de proteína y kilogramos totales de proteína no se encontraron diferencias significativas $(\mathrm{p} \geq 0,05)$ durante el transcurso de la lactancia para los genotipos estudiados. Se evidenciaron valores que oscilaron entre 3,2 y 3,3\% para los primeros 150 días de lactancia, superiores a los $2,7 \%$ y $2,7 \%$ en promedio por lactancia para la raza Holstein y Normando o los 2,8 a $2,9 \%$ en vacas Holstein, Brown Swiss, Normando y sus cruzas ${ }^{15}$, coincidiendo con los porcentajes 3,1 a 3,4 encontrados para la raza Holstein ${ }^{2,10,16}$. Los factores más influyentes en la concentración de proteína en la leche son la genética y la alimentación, siendo el primero de ellos el más importante, existiendo además otras fuentes de variación como edad, sanidad de la ubre y etapa de lactación ${ }^{8}$.

En el presente trabajo las edades al primer parto (en meses) fueron: B $(37,1 \pm 1), \mathrm{J}(39,7 \pm 1), \mathrm{S}(42,6 \pm 1)$ y $\mathrm{G}(41,4 \pm 1)$, más altas que las halladas en otras investigaciones, que reportan $34,5 \pm 5$ meses para este parámetro $^{5}$. Al respecto, se asevera que las ganancias de peso comprendidas entre los 600 y 750 gramos por día serían óptimas para las futuras vacas lecheras y que valores menores podrían retrasar la pubertad ${ }^{18}$.

Sin embargo, en general se trata de trabajos que no han sido realizados en ambientes como el de la amazonia, por lo que la transferencia de los resultados derivados de ellos no resulta siempre posible. A pesar de que las vaquillonas en pastoreo podrían seleccionar el forraje que consumen, los pastos utilizados con frecuencia no alcanzan a proveer los nutrientes requeridos, especialmente en términos energéticos (pasturas templadas con promedio de energía digestible 2,5 Mcal/ $\mathrm{Kg} / \mathrm{MS}$ y pasturas tropicales con $1,4 \mathrm{Mcal} / \mathrm{Kg} / \mathrm{MS}$ ). La alta competitividad de este sistema está relacionada con el patrón estacional de crecimiento de las especies forrajeras utilizadas, que dependen básicamente de una apropiada humedad, lluvias y fertilidad del suelo. Esto podría explicar en parte la razón de tan elevada edad al primer parto, reafirmando las características propias de los sistemas a pastoreo libre, en los que se hace difícil mantener una ganancia de peso diaria sostenida, ya que se depende del factor ambiental y calidad de los forrajes.

Aquí, los pesos al parto de cada uno de los genotipos distaron del óptimo establecido para cada cruzamiento, posiblemente por la limitación impuesta por las condiciones ambientales y nutricionales. Estas mismas vacas registraron los siguientes aumentos de peso diario ( $\mathrm{kg}$ promedio $\pm \mathrm{EE}$ ) hasta el parto: $\mathrm{G}(0,190 \pm 0,03)$, $\mathrm{J}(0,230 \pm 0,01), \mathrm{B}(0,240 \pm 0,02)$ y S $(0,220 \pm 0,01)$. El mejor genotipo en términos de velocidad de crecimiento fue el de las vacas B, al que le correspondió comparativamente el peor ambiente. Los resultados obtenidos muestran que el genotipo más pesado (B) fue el que perdió menos $\mathrm{kg}$ de peso desde el parto al secado, más allá de que no recuperó su peso al parto. Es difícil determinar la causa de esta situación, pero evidentemente podría ser un indicador de mejor adaptación a las condiciones de la amazonia.

La eficiencia reproductiva es un reto para las ganaderías y los productores de leche en el mundo. Uno de los principales problemas en los hatos es el retraso en el reinicio de la actividad ovárica después del parto ${ }^{6}$. Se encontraron valores de $452 \pm 100$ días de intervalo parto-parto en vacas de doble propósito basado en el mestizaje de razas cebuínas con Holstein ${ }^{12}$, el cual es menor a los encontrados en este trabajo. No obstante, es contradictorio a los guarismos encontrados para el intervalo parto-primer celo, donde los valores están dentro del óptimo buscado para animales especializados y en ambientes más benignos, al igual que para los valores del intervalo parto-concepción. Ello indicaría que la posibilidad de llevar adelante una gestación estaría limitada.

Es conocido que las vacas lecheras poseen requerimientos de energía vinculados especialmente con su mantenimiento y sus niveles de producción. O sea, cuanto más pesada sea la vaca, mayores requerimientos de mantenimiento se deberán satisfacer $\mathrm{y}$, ante aportes limitados de alimento, podría derivar menos energía para producir leche e incluso podrían verse afectados sus parámetros reproductivos.

Los resultados obtenidos coinciden con trabajos que demuestran que el comportamiento productivo y reproductivo de los genotipos y sus cruzas parecen estar muy influenciados por la disponibilidad de nutrientes, ya que en condiciones de mayor calidad y cantidad de alimentos concentrados, el genotipo más especializado superaría en producción de leche a las cruzas, pero al mismo tiempo sería el genotipo más afectado cuando existan restricciones alimenticias, situación similar a lo acontecido para todas las cruzas en el actual estudio.

Se concluye que los cuatro genotipos lecheros estudiados se comportan de forma similar acorde a la región en estudio. Ninguno de los genotipos lecheros analizados se destacó por encima del otro, mostrando una paridad en este aspecto. 


\section{REFERENCIAS}

1. Acosta J, Padrón S, Pereira N, Rincón E, Chirinos Z, Villalobos R, Marin D. 1998. Producción de leche de ganado mestizo en una zona de bosque seco tropical. Rev Cientif FCV-LUZ 4: 99-104.

2. Alvarez HJ. 2006. Producción de leche bovina con distintos niveles de asignación de pastura y suplementación energética. Cien Inv Agr 33: 99-107.

3. Buckley F, Lopez N, Heins BJ. 2014. Crossbreeding: implications for dairy cow fertility and survival. Animal 8: 122-133.

4. Duran VC, Duran JM, Suárez JF, Molina EJ, Molina CH. 2009. La raza colombiana Lucerna: una alternativa para la producción de leche y carne en medio de sistemas. En: Producción Animal en la Amazonia Colombiana, Edit. Universidad de la Amazonia, Florencia (Colombia), 184 p.

5. Freitas AF, Costa CN, Menezes CR. 2008. Programa de melhoramento da raça Girolando-teste de progênie: sumario de touros, Publ. Universidade Federal de Juiz de Fora, Minas Gerais, Brasil, p. 20.

6. Guáqueta HM, Zambrano JV, Jiménez CE. 2014. Risk factors for ovarian postpartum resumption in Holstein cows, under high tropical conditions. Rev Med Vet Zoot Córdoba 19: 3970-3983.

7. Instituto Nacional Autónomo de Investigaciones Agropecuarias (INIAP). 1997. Manual de pastos tropicales para la Amazonia Ecuatoriana, Manual № 77 , Guayaquil. On line: http://repositorio.iniap.gob.ec/bitstream/41000/1622/1/Manual\%20n \%C2\%BA\%2011\%20 de\%20pastos\%20tropicales\%20reducido\%20ultimo.pdf

8. Kruze J, Latrillé L. 1999. Calidad higiénica de la leche cruda en Chile. Avan Prod Anim 1: 107-139.

9. Madalena FE. 2012. Animal breeding and development; South American perspective. J Anim Breed Genet 129: 171-172.
10. Mayorga MO. 2011. Evaluación de sistemas de alimentación en vacas Holstein y su efecto sobre la productividad animal, la emisión de metano y de óxido nitroso y la captura de carbono en la Sabana de Bogotá. Publ. Corp. Colomb. Inv. Agropec. http://www.scielo.org.ve/ scielo.php?script $=$ sci_nlinks\&ref $=2954076 \&$ pid $=$ S0798$7269201500010000300020 \& \operatorname{lng}=\mathrm{es}$

11. Molina-Echeverri JJ. 2009. Manejo reproductivo en ganaderías de doble propósito. En: Producción Animal en la Amazonia Colombiana, Edit. Universidad de la Amazonia, Florencia (Colombia), p. 28-32.

12. Motta PA, Rivera LG, Mariño AA. 2012. Productive and reproductive performance of F1 Gyr x Holstein cows in Colombian warm climate. Vet Zootec 6: 17-23.

13. Román-Ponce SI, Ruiz FJ, Montaldo H, Rizzi R, Román H. 2013. Efectos de cruzamiento para producción de leche y características de crecimiento en bovinos de doble propósito en el trópico húmedo. Rev Mex Cienc Pec 4: 405-416.

14. Román-Ponce SI. 2015. Mejoramiento genético de los bovinos del trópico. En: Estado del arte sobre investigación e innovación tecnológica en ganadería bovina tropical, Publ. Conacyt, Capit. IV, México, p. 99-153.

15. Rodriguez CE, Saavedra GF, Gómez DF. 2015. Efecto de la etapa de lactancia sobre la calidad fisicoquímica de leche en vacas de raza Holstein y Normando. Zoot Trop 33: 23-35.

16. Roca-Fernández AI et al. 2009. Efecto de la raza de vaca y del nivel de suplementación sobre la producción y calidad de leche en pastoreo. Investig Agr 1: 391-398. http:// ciam.gal/uploads/publicacions/732archivo

17. Vargas B, Ulloa J. 2008. Relación entre crecimiento y curvas de lactancia en grupos raciales lecheros de distintas zonas agroecológicas de Costa Rica. On line: http:// www.lrrd. org/lrrd20/8/varg20122.htm

18. Wathes DC, Pollott GE, Johnson KF, Richardson H, Cook JS. 2014. Heifer fertility and carry over consequences for life time production in dairy and beef cattle. Animal 8: 91-104. 